\title{
RELATÓRIO DA TESOURARIA — PERÍODO DE 1978/1979
}

* Taka Oguisso

RBEn/09

OGUISSO, T. - Relatório da tesouraria - período de 1578/1979. Rev. Bras. Enf,; DF, $32: 438-446,1979$.

A Tesouraria constitui, geralmente, um reflexo do estado de uma instituição. A Associação Brasileira de Enfermagem em suas épocas heróicas não podia ser avaliada em termos de realizaçōes apenas pela verificação de seus balanços e balancetes. Isso porque os membros, que sucessivamente ocupavam os cargos de Diretoria, se revezavam em suas cotas de sa.crificio, realizando muito mais que os recursos permitiam. Essa tradiçāo de traballıo foi a herança deixada pelas que nos antecederam nos cargos que hoje ocupamos. Entretanto, a própria evoluçāo social e a criação ou organização de setores específicos para regulamentação e controle de atividades de instituiçōes de finalidade cientifica ou cultural, tornam a situação atual muito diferente daquela vivida há apenas cinco ou dez anos atrás pela ABEn. A cada dia surgem novas posturas e exigências legais que obrigam a ABEn a se organizar como uma verdadeira empresa, se quiser viver e sobreviver a todas essas determinaçōes legais, fiscalizaçōes e diligências.
Apesar de ainda se realizar muita coisa graças à boa vontade e senso de responsabilidade pelo cargo assumido, observa-se pelo movimento da Tesouraria e principalmente pelo orçamento-programa que toda atividade tem um preço e necessita de uma fonte de recursos. t nesse sentido que a Tesouraria constitui um instrumento de medida do estado da entidade e de suas realizaçōes. $O$ que importa não é fazer a mágica de realizar grandes obras sem recurso, mas adminstrar bem o recurso existente, aplicando-o em programas prioritários, assim decididos pela Assembléia de Delegados, obtendo a máxima rentabilidade possível. Não pode ser esquecido também que essa administração também exige recursos para sua própria manutenção. Há que se incluir os custos para conservação do prédio, o material, o pessoal, taxas, impostos, utilidades públicas, reuniões de Diretoria, parcelas das despesas para representação da ABEn em organizaçōes internacionais, etc.

A "Casa da Enfermeira" que tem conta bancária exclusiva destinada a

(*) 1. Tesoureira da ABEn - quadriênio 1976-1980. 
OGUISSO, T. - Relatório da tesouraria - período de 1978/1979. Rev. Bras. Enf,; DF, 32 : 438-446, 1979.

criar fundos para construção estava em 1978 com Cr\$90.199,76 (noventa mil, cento e noventa e nove cruzeiros e setenta e seis centavos). Recebeu nova injeção de recursos com o depósto de mais $2 \%$ sobre a receita de 1978, no valor de $\mathrm{Cr} \$ 41.711,68$ (quarenta e um mil, setecentos e onze cruzeiros e sessanta e oito centavos). Com os juros e correção monetária está atualmente com Cr\$ .... 117.991,00 (cento e dezessete mil, novecentos e noventa e um cruzeiros).

Entre as despesas de maior vulto da ABEn continua sendo a aquisição de papel e impressão da Revista Brasileira de Enfermagem, que custou Cr\$505.308,20 (quinhentos e cinco mil, trezentos e oito cruzeiros e vinte centavos).

Os Anais dos Congressos de Enfermagem continuam a ser subsidiados por verbas de doação obtidas por intermédio da Coordenadora da Comissão de Divulgação e Publicações, Dr. ${ }^{a}$ Lygia Paim, junto à Secretaria do Ensino Superior, antigo Departamento de Assuntos Universitários, do Ministério da Educação e Cultura.

Outra despesa elevada no período foi a montagem do auditório da ABEn, o que foi feito com a receita obtida no XXX Congresso Brasileiro de Enfermagem. Essa montagem, cujo total atingiu a $\mathrm{Cr} \$$ 332.783,63 (trezentos e trinta e dois mil, setecentos e oitenta e três cruzeiros e sessenta e três centavos), consistiu na aquisição de mobiliário, equipamentos e decoração do salāo localizado no pavimento térreo da sede da ABEn, em Brasília.

Em prosseguimento ao estudo iniciado no ano passado sobre crescimento e evolução das Seções foi elaborado o Quadro Demonstrativo constante do Anexo I. Esse estudo abrange o período de cinco anos, de 1974 a 1978. A Seção RJ inclui as antigas Seções Rio de Janeiro e Guanabara, apesar de só em 1976 elas tiverem sido fundidas. Os números de associados dessas Seções foram somados, inclusive o do Distrito de Volta Redonda.
Em 1978, pela primeira vez na história da $\mathrm{ABEn}$, foi atingido 0 total de 5.376 associados, o que corresponde a 43\% do total de 12.381 enfermeiros e obstetrizes registrados no Conselho Federal de Enfermagem até dezembro desse mesmo ano.

Entretanto, considerando que podem ser associados da ABEn enfermeiros, obstetrizes, técnicos de enfermagem e estudantes do curso de graduação matriculados nos dois últimos períodos, o percentual, na verdade fica em torno de $40 \%$ ou menos, pois os técnicos de enfermagem são incluídos como associados efetivos, porque as relações nominais em 1978 não previam essa distinção. O total nesse ano foi de 5.000 efetivos e 376 associados especiais ou estudantes de enfermagem.

A Seção que conta com maior número de associados é São Paulo com 1.125 asscoiados quites e a menor é Espírito Santo com 25. A Seção que mais cresceu foi Mato Grosso, que tinha 10 (dez) associados em 1974 e está com 56 em 1.978. Apresentaram também crescimento significativo as Seções: Bahia, Alagoas, Santa Catarina, Rio Grande do Norte, Paraíba, Pará, Minas Gerais e Piauí, todas com índice superior a $100 \%$ do número de sócios de 1974 em relação ao ano de 1978. Apenas duas Seções: Distrito Federal e Espírito Santo tiveram menos sócios em 1978 do que em 1974.

No conjunto, a ABEn cresceu $68,6 \%$ de 1974 a 1978.

A Seção Pará que permanecia na faixa de 60 a 80 associados anuais passou a $194(142,5 \%)$ em 1978, em conseqüência de ter sido sede de Congresso, o que mais uma vez comprova que esse tipo de evento é altamente benéfico para a Seção.

Cotejando apenas os dados de 1977 e 1978 verifica-se que quase todas as Seções cresceram. Esse fenômeno não ocorreu apenas na Seção Paraná, que teve menos associados em 1978 do que ano anterior. Duas Seções encontram-se es- 
OGUISSO, T. - Relatório da tesouraria - período de 1978/1979. Rev. Bras. Enf,; DF, 32 : 438-446, 1979.

tacionárias: Amazonas e Espírito Santo, que tiveram exatamente o mesmo número de sócios nos dois anos considerados. Aliás, a Seção Espírito Santo, desde que foi reativada em 1974, não conseguiu se desenvolver, necessitando talvez de maior ajuda da ABEn Central, pois até mesmo a correspondência tem retornado.

Algumas Seçōes merecem destaque especial por terem crescido sem retrocesso algum nos últimos cinco anos. São elas: Ceará, Rio Grancie do Norte, Paraíba, Alagoas, Mato Grosso e Santa Catarina.

O Anexo II apresenta o Quadro Demonstrativo da evolução da ABEn entre 1977 e 1978, relacionando dados quanto ao número de sócios efetivos e especiais. A data de 31 de maio é demarcatória de alteração do valor de "per capita", o đue implica também na alteração do valor global do "per capita" devido. Este é o o produto da multiplicação do número de sócios com o valor "per capita" correspondente. Teoricamente, a ABEn deveria

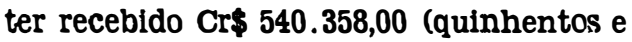
quarenta mil e trezentos e cinqüenta e oito cruzeiros como receita provida das Seçōes a título de "per capita". O valor constante no Demonstrativo da Receita e Despesas de janeiro e dezembro de 1978 é de Cr\$547.862,00 (quinhentos e quarenta e sete mll, oitocentos e sessenta e dois cruzeiros) porque as Seçōes costumam pagar o último trimestre e às vezes até $o$ penúltimo trimestre também no ano seguinte. Daí a diferença nos dois valores.

O Anexo III apresenta a situação referente ao número de associados quites em 197.9, durante o primeiro semestre, por Seção e Distrito, e respectivo "per capita" recebido. Algumas Seções e Distritos remeteram o numerário mas não enviaram a relação nominal de sócios quites, como ocorreu com a Seção Minas Gerais e os Distritos de Caxias do Sul e Santa Maria do Ro Grande do Sul. Outras Seçōes remeteram valor superior ao devido de acordo com a relação nominal como ocorreu com as Seções: Maranhão, Rio Grande do Norte, Paraíba, Alagoas e Mato Grosso.

Entretanto, essas situações são provisórias e serão regularizadas durante o Congresso, através de contatos pessoais com as Tesoureiras das Seçōes como tem ocorrido anteriormente.

Como se vê, a maioria das Seções procurou remeter o "per capita" dentro do prazo estabelecido, beneficiando-se do valor sem a majoração de $10 \%$.

O Anexo IV apresenta o Quadro Demonstrativo de balanços e balancetes mensais recebidos e não recebidos tas Seçōes e Distritos. Como medida de unificação de contabilidade, pelo menos à nível de Seção, esta fol orientada a fundir o seu balancete com o do Distrito, razão que deve estar difiçultando a remessa desses documentos, como se pode verificar pelo Quadro. As Seçōes Pernambuco, Minas Gerais, Goiás, Espírito Santo e Santa Catarina não remeteram os balancetes mensais, o Demonstrativo da Receita e Despesas do ano de 1978 e nem o Balanço Patrimonial, Seria por demais ocioso repetir o que vem sendo explicado ano a ano sobre a importância desses documentos para a Tesouraria compor o balanço anual da ABEn. Por isso, fica consignado apenas que a ABEn como uma verdadeira empresa está sujeita aos dispositivos legais citados no início e as Tesoureiras, assim como as Diretorias das Seções e da ABËn Central, terão que assumir a responsabilidade por esse estado de coisas, ocorrância essa já registrada recentemente.

Quanto ao estudo sobre a participação da ABEn em organizaçōes internacionais, esta Tesouraria que se propôs a realizá-lo, com aprovação da Diretoria, vem comunicar as conclusões.

De acordo com os objetivos traçacios, o estudo de uma amostra constituída de 85\% de uma população de 1.325 participantes que compareceram ao XXX Congresso Brasileiro de Enfermagem, em Belém, do Pará, possibilita extrair as seguintes conclusōes: 
OGuIsso, T. - Relatório da tesouraria - período de 1578/1979. Rev. Bras. Enf;; DF, 32 : 438-446, 1979.

1) A grande maioria dos associados, em geral, isto é, $72,4 \%$, considera importante a filiação da ABEn às entioades internacionas: Conselho Internaciunal de Enfernieiras (CIE), Comitê Internacional Catciico de Enfermeiras e Assistentes Médi:o-Sociais (CICIAMS) e $\overrightarrow{r e}$ deração Panamericana de Enfermeiras/ os (FEPAEN). Se considerados apenas os ásscciados oue já ocuparam cargo de dlretoria cia ABEn, esse percentual aumerita pira 31,3\%

2) O exercício de cargo de dirctoria da ABEn favoreec a maior partıcipação do associado em congressos, tanto em nacionnais promovidos pela ABEn como em internacionais promovidos pe!ns enticiades internacionais referidas.

3) Os cargos de diretoria da ABEn sāo preenchidos principalmente por associados cuja principal função encontrase na área de ensino. Entretanto, sāo os profissionais oriundos da área hospitalar que constituem a maior clientela que freqüenta os congressos de enfermagem.

4) Quanto à época de formação profissional, observa-se que enfermeiros formados até 1970 são mais favoráveis à fillaçāo mediante contribuição adicional ao CICIAMS, enquanto enfermeiros formados após 1971, são favoráveis ao CIE.

5) Apesar de $35,7 \%$ dos congressistas que devolveram o questionário não terem feito opção para nenhuma organização internacional (a maioria dos associados, isto é, $55,8 \%$ do total de 1.127 participantes opinaram pela manutenção de filliaçāo da ABEn a entidades internacionais. Apenas 8,5\% manifestaram-se desfavoravelmente. A entidade mais votada pelos congressistas fol o CIE, seguida pelo CICIAMS e em terceiro lugar a
FEPAEn). Do total de 629 congressistas que optaram para uma ou mais entidades, $141(22,4 \%)$ são integrantes ou exintegrantes de diretoria da ABEn, o que vale dizer, são líderes na enfermagem, eleitos por seus pares, e portanto elementos representativos da classe e do pensamento dos associados, em geral.

A luz dos preceitos estatutários nāo só da $A B E n$, mas principalmente das entidades internacionais às quais está fíliada; do principio de coerência interna que deve ser resguardado quanto ao crescimento da Associação e obrigaçōes correspondentes; e ainda, o principio da unidade que deve ser mantido na ABEn para assegurar sua continuidade como organizaçāo forte e livre, verifica-se que a experiência feita nas décadas de 195060 , solicitando-se ao associado para declarar, por escrito, a que setor ou setores internacionais desejava pertencer, pagando uma cota suplementar, poderia constituir, talvez solução para o caso do CICIAMS, mas não para o CIE.

Há que se reiterar que o membro da ABEn não pode isoladamente pertencer ou pedir filliação a essas entidades internacionais. a ABEn que irá se filliar, ou não. E ABEn são todos os associados quites com a Tesouraria.

A soluçāo correta e ideal, pois, seria declarar o número exato de associados às entidades internacionais e arcar com o ônus dessa fillação, ou então, assumir o desligamento. Essa hipótese, por sinal, já fol tentada, sem êxito, diversas vezes. Por fím, a própria ABEn acabou desistindo de solicitaçōes de desligamento.

O ónus da fillação seria para 1978 , calculado ao câmbio do dia 26/04/79, para 5.000 associados efetivos o seguinte:

$\begin{array}{ll}\text { Para o ICN } & 5.000 \times \text { SF } \$ 2,20 \text { ou } \\ & 5.000 \times \$ 30,60=\$ 153.000,00 \\ \text { Para o CICIAMS } & 5.000 \times \text { BF } \$ 13,25 \text { ou } \\ & 5.000 \times \$ 10,51=52.550,00 \\ \text { Para a Federação }- & \text { US } \$ 600 \text { ou } \\ & \$ 23,79 \times 600=\$ 14.274,00\end{array}$

TOTAL para as três Organizaçōes Internacionais $=\mathbf{C r} \$ \mathbf{2 1 9} . \mathbf{8 2 4 , 0 0}$. 
OGUISSO, T. - Relatório da tesouraria - período de 1978/1979. Rev. Bras. Enf,; DF, 32 : 438-446, 1979.

A aparente irrelevância do objeto deste estudo adquire aqui um certo vulto, pois trata-se de decidir uma questão que terá repercussões em, pelo menos, oitenta e sete paises, onde o CIE tem associações nacionais filiados. A ABEn já enfrentou crises muito mais difíceis, nas memoráveis fases heróicas, em que os membros de diretoria se revezavam nas cotas de dedicação e sacrifício para manter acesa a chama de unidade e de vida da ABEn.

Também o CICIAMS, do qual é membro desde 1955, isto é, há vinte e quatro anos, dado o número de profissionais de enfermagem de religião católica - é um caso que merece muita reflexão. A ABEn tem um grande débito de gratidão às religiosas que, em épocas passadas, recentes e atuais, vem contribuindo para o crescimento da Associação. A história da ABEn registra inúmeros nomes de religiosas, como Madre Marie Domineuc, Irmã Matilcle Nina, Irmã Maria Gabriela Nogueira, Madre Maria Aurea da Cruz e a própria Irmã Maria Thereza Notarnicola, nossa atual Secretária Executiva. O Brasil já foi sede de dois congressos internacionais do CICIAMS e vem participando de outros, realizados em diferentes países, enviando representantes, como recentemente na Costa do Marfim, na Africa.

Quanto à FEPAEn, de instituição recente e filiação da ABEn há apenas nove anos, não representa o maior encargo, por enquanto, porque a taxa anual é um valor fixo. Sendo uma entidade que congrega associações dos países latino-americanos, a representação do Brasil, pela sua dimensão territorial e populacional, inclusive quanto ao número de profissionais formados e em exercício, constitui certamente o maior núcleo associativo da enfermagem. Insistentemente tem sido solicitado que a ABEn assuma a direção e realize um congresso da FEPAEn.

A falta de maior divulgação sobre a matéria entre os associados de um modo geral, a talvez maior correspondência com essas entidades, além de maior participação da ABEn em reuniões promovidas por essas entidades, têm gerado desconhecimento das responsabilidades da ABEn como membro dessas organizações.

Porém, tudo isso constitui um pesado encargo financeiro que os "per capita" enviados pelas Seções não tem conseguido cobrir. Por outro lado, anuidades elevadas cificultam o recrutamento de associados, principalmente depois da criação e implantação dos Conselhos de Enfermagem, onde a inscrição e o pagamento de anuidade são compulsórios.

Embora o Conselho de Enfermagem constitua um sinal visível da evolução da enfermagem no País, assim como a criação dos Sindicatos de Enfermeiros, ambos frutos do empenho e esforço da própria ABEn, é possível que o nível de conscientização profissional e associativo dos enfermeiros, obstetrizes e técnicos de enfermagem ainda não tenha atingido suficiente maturidade para compreender a necessidade das três instituições, cada uma, com seus objetivos distintos e bem definidos.

Talvez no futuro, a fórmula ideal seja o entrosamento, quem sabe até a integração, da ABEn com o Conselho de Enfermagem, para unidos e fortalecidos, estudarem mais profundamente o problema da filiação às entidades internacionais, inclusive verificando como as associações de enfermagem de outros países solucionam o problema. 
OGuisso, T. - Relatório da tesouraria - período de 1978/1979. Rev. Bras. Enf,; DF, $32: 438-446,1979$.

ANEXO I

QUADRO DEMONSTRATIVO DE SOCIOS QUITES DA ABEN, POR SEÇAO, DE 1974-1978, EM NUMMEROS ABSOLUTOS E PORCENTAGEM

\begin{tabular}{|c|c|c|c|c|c|c|c|c|c|c|c|}
\hline Seção & 1974 & 8 & 1975 & 8 & 1976 & 8 & 1977 & 8 & 1978 & 8 & $\begin{array}{l}\text { Percontual de } \\
\text { cresclenerio }\end{array}$ \\
\hline AYY & 88 & 2,8 & 95 & 2,4 & 89 & 2,0 & 100 & 2,2 & 100 & 1,9 & 13,6 \\
\hline $\mathrm{Pl}$ & 80 & 2,5 & 60 & 1,5 & 74 & 1,6 & 80 & 1,8 & 194 & 3,6 & {$[42,5$} \\
\hline $\mathrm{CE}$ & 113 & 3,5 & 123 & 3,2 & 183 & 4,1 & 203 & 4,5 & 205 & 3,8 & 31,4 \\
\hline MA & 58 & 1,8 & 99 & 2,6 & 110 & 2,4 & 86 & 1,9 & 111 & 2,0 & $4: 8$ \\
\hline PI & 32 & 1,0 & 33 & 0,9 & 52 & 1,2 & 41 & 0,9 & 70 & 1,3 & $=1 \theta, 8$ \\
\hline $\mathrm{RN}$ & 30 & 0,9 & 31 & 0,8 & 42 & 0,9 & 60 & 1,3 & 79 & 1,5 & $=83,3$ \\
\hline$P E$ & 86 & 2,7 & 163 & 5,1 & 295 & 6,6 & 108 & 2,4 & 161 & 3,0 & $8\}, 2$ \\
\hline$F B$ & 81 & 2,6 & 82 & 2,1 & 127 & 2,8 & 193 & 4,3 & 198 & 3,7 & $2 A, 4,4$ \\
\hline SII & 17 & 0,5 & 21 & 0,5 & 31 & 0,6 & 38 & 0,8 & 55 & 1,0 & 223,6 \\
\hline SE & 19 & 0,6 & 18 & 0,4 & 40 & 0,8 & 32 & 0,7 & 43 & 0,8 & 126,3 \\
\hline I $A$ & 118 & 3,7 & 244 & 6,4 & 258 & 5,7 & 237 & 5,3 & 447 & 8,3 & 278,8 \\
\hline$m$ & 10 & 0,3 & 23 & 0,6 & 32 & 0,7 & 32 & 0,7 & 56 & 1,0 & 460,0 \\
\hline x.G & 164 & 5,1 & 186 & 4,8 & 330 & 7,3 & 316 & 7,3 & 366 & 6,8 & 123,2 \\
\hline Go & 103 & 3,2 & 192 & 5,0 & 165 & 3,7 & 145 & 3,2 & 155 & 2,8 & 50,4 \\
\hline$D P$ & 407 & 12,8 & 366 & 9,5 & 299 & 6,7 & 339 & 7,5 & 381 & 7,1 & $-6,4$ \\
\hline BJ & 765 & 23,9 & 861 & 22,5 & 1033 & 22,9 & 913 & 20,4 & 1036 & 19,3 & 35,4 \\
\hline $\mathbb{S P}$ & 666 & 20,9 & 826 & 21,6 & 802 & 17,9 & 1018 & 22,7 & 1125 & 21,0 & 68,8 \\
\hline $\mathrm{PR}$ & 162 & 5,1 & 147 & 2,8 & 131 & 2,9 & 240 & 5,4 & 230 & 4,3 & 42,0 \\
\hline sc & 54 & 1,7 & 76 & 1,9 & 106 & 2,4 & 111 & 2,5 & 151 & 2,8 & 179,6 \\
\hline BS & 109 & 3,4 & 149 & 2,8 & 275 & 6,1 & 173 & 3,9 & 188 & 3,5 & 72,5 \\
\hline ES & 27 & 0,9 & 27 & 0,7 & 27 & 0,6 & 25 & 0,6 & 25 & 0,5 & $-7,4$ \\
\hline TOTAT & 3189 & 100,0 & 3822 & 100,0 & 4501 & 100,0 & 4489 & 100,0 & 5376 & 100,0 & 68,5 \\
\hline
\end{tabular}

Percentual de crescimento de 1974 a 1978.

Fonte - Tesouraria da ABEn. 
OGuIsso, T. - Relatório da tesouraria - período de 15.78/1979. Rev. Bras. Enf,; DF, 32 : 438-446, 1979.

\section{ANEXO II}

QUADRO DEMONSTRATIVO DE SOCIOS QUITES DA ABEn EM 1977 E 1978, POR SEÇĀO, E "PER CAPITA" DEVIDO EM 1978

\begin{tabular}{|c|c|c|c|c|c|c|c|c|c|}
\hline \multirow{2}{*}{ sietespe } & \multicolumn{3}{|c|}{1977} & \multicolumn{4}{|c|}{1978} & \multirow[b]{2}{*}{ Total } & \multirow{2}{*}{$\begin{array}{l}\text { "Per oapita" } \\
\text { devido } \\
\text { con os }\end{array}$} \\
\hline & $\begin{array}{l}\text { Rfet. } \\
\$ 100\end{array}$ & $\begin{array}{l}\text { Bsp. } \\
860\end{array}$ & Total & $\begin{array}{l}\text { sfet. } \\
\$ 100\end{array}$ & $\begin{array}{l}\text { Esp. } \\
860\end{array}$ & $\begin{array}{l}\text { Bfet. } \\
\text { s110 }\end{array}$ & $\begin{array}{l}31 / 7 \\
\text { Eep. } \\
866\end{array}$ & & \\
\hline AR & 100 & - & 100 & 40 & - & 53 & 7 & 100 & 10.292 \\
\hline $\mathbf{P A}$ & 80 & - & 80 & 96 & 7 & 73 & 18 & 194 & 19.238 \\
\hline G2 & 203 & - & 203 & 56 & - & 149 & - & 205 & 21.990 \\
\hline $\mathbf{m a}$ & 86 & - & 86 & 23 & - & 88 & - & 111 & 11.980 \\
\hline PI & 42 & - & 41 & 34 & 17 & 17 & 2 & 70 & 6.422 \\
\hline PO & 60 & - & 60 & - & - & 79 & - & 79 & 8.690 \\
\hline PB & 108 & - & 108 & 42 & - & 119 & - & 16 & 17.290 \\
\hline $\mathbf{P B}$ & 193 & - & 193 & 130 & 32 & 27 & 9 & 198 & 18.484 \\
\hline ar & 38 & - & 38 & 14 & - & 29 & 12 & 55 & 5.382 \\
\hline $\mathbf{S T}$ & 31 & - & 31 & 20 & - & 23 & - & 43 & 4.530 \\
\hline $\mathrm{BA}$ & 231 & 6 & 237 & 107 & 16 & 253 & $\pi$ & 447 & 44.176 \\
\hline $\mathbf{r r}$ & 32 & - & 32 & 19 & - & 20 & 17 & 56 & 5.221 \\
\hline W & 316 & - & 316 & 322 & - & 44 & - & 366 & 37.040 \\
\hline GO & 145 & - & 145 & 27 ". & - & 128 & - & 155 & 16.780 \\
\hline DP & 339 & - & 339 & 369 & - & 12 & - & 381 & 38.220 \\
\hline RJ & 913 & - & 913 & 767 & 1 & 253 & 15 & 1036 & 105.580 \\
\hline $\mathbf{S P}$ & 1018 & - & 1018 & 643 & - & 800 & 82 & 1125 & 113.712 \\
\hline $\mathbf{P R}$ & 192 & 48 & 240 & 121 & 47 & 57 & 5 & 230 & 21.520 \\
\hline sc & 96 & 15 & 111 & - & - & 133 & 18 & 151 & 15.818 \\
\hline BS & 173 & - & 173 & 53 & - & 135 & - & 188 & 20.150 \\
\hline ES & 25 & - & 25 & 9 & - & 16 & - & 25 & 2.660 \\
\hline Total & 4420 & 69 & 4489 & 2892 & 120 & 2108 & 256 & 5376 & 545.065 \\
\hline
\end{tabular}

Fonte - Tesouraria da ABEn. 
OGUISSO, T. - Relatório da tesouraria - período de 1978/1979. Rev. Bras. Enf,; DF, $32: 438-446,1979$.

\section{ANEXO III}

QUADRO DEMONSTRATIVO DE SÓCIOS EM 1979, ATE 11-8, POR SEÇAO E DISTRITO E RESPECTIVO "PER CAPITA"RECEBIDO

\begin{tabular}{|c|c|c|c|c|c|c|c|c|c|}
\hline $\begin{array}{c}\text { See } \\
\text { çào }\end{array}$ & $\begin{array}{l}\text { D15 } \\
\text { tri } \\
20\end{array}$ & $\begin{array}{c}\text { So } \\
\text { até } 3 \\
\text { Efet. }\end{array}$ & $\begin{array}{l}\text { I0 S } \\
.5 \\
\text { Esp. }\end{array}$ & $\begin{array}{l}\text { QDI } \\
\text { após } \\
\text { Blet. }\end{array}$ & $\begin{array}{c}\text { T B S } \\
31.5 \\
\text { Bsp. }\end{array}$ & TOTAL & $\begin{array}{l}\text { Per appita } \\
\text { recobido } \\
\text { em as }\end{array}$ & $\begin{array}{l}\text { Débito } \\
\text { em as }\end{array}$ & $\begin{array}{l}\text { Crédito } \\
\text { ets of }\end{array}$ \\
\hline AS & & 43 & - & 33 & 3 & 79 & 5.590 & 5.016 & \\
\hline$P A$ & & - & - & 129 & 21 & 150 & 20.064 & 352 & \\
\hline CB & & 138 & - & 270 & 125 & 533 & 65.830 & 3.095 & \\
\hline eA & & 50 & - & 15 & - & 65 & 9.645 & & 1.000 \\
\hline PI & & 18 & 3 & 34 & 54 & 109 & 12.675 & 152 & \\
\hline For & & 48 & - & 20 & 3 & 71 & 9.734 & 267 & \\
\hline PB & & 53 & - & 112 & - & 165 & 21.905 & 1.001 & \\
\hline PB & & 126 & 24 & 43 & 87 & 280 & 31.966 & 1.336 & \\
\hline Al & & 25 & 22 & 23 & 14 & 84 & 9.806 & 99 & \\
\hline ss & & 25 & 11 & 27 & 1 & 64 & 5.405 & 2.795 & \\
\hline $\mathrm{BA}$ & & 39 & 1 & 180 & 32 & 252 & 33.264 & 804 & \\
\hline MT & & 10 & - & 20 & 1 & 31 & $4 \cdot 340$ & & 895 \\
\hline Gis & & 135 & - & 114 & 3 & 252 & 34.105 & 44 & \\
\hline 10 & J.F. & 93 & - & 7 & - & 100 & 13.091 & & \\
\hline GO & & 43 & - & 54 & 11 & 108 & 14.401 & & \\
\hline DP & & 338 & - & - & - & 338 & 43.940 & & \\
\hline BS & & - & - & $\cdots$ & - & $\cdots$ & - & & \\
\hline BJ & R.J. & 515 & 4 & 90 & 5 & 614 & 67.310 & 13.365 & \\
\hline R.J & Mit. & 67 & 14 & 49 & 8 & 138 & 17.309 & 460 & \\
\hline$R J$ & V.R. & 19 & - & 8 & - & 27 & 3.614 & & \\
\hline SP & S.P. & 631 & 35 & 56 & 2 & 724 & 93.386 & & \\
\hline SP & Camp & 96 & 6 & - & - & 102 & 13.020 & & \\
\hline$S P$ & Sant & - & - & 13 & - & 13 & 1.859 & & \\
\hline SP & R.P. & 58 & - & - & - & 58 & 7.540 & & \\
\hline$S P$ & Arar. & - & - & 49 & 13 & 62 & 8.294 & & \\
\hline FR & cur. & 76 & 5 & 23 & 15 & 119 & 15.104 & & \\
\hline$P R$ & Lond. & - & - & 37 & - & 37 & 5.291 & & \\
\hline sc & & 117 & 34 & 9 & 2 & 162 & 18.270 & 1.485 & \\
\hline RS & P.A. & 72 & - & - & - & 72 & 9.360 & & \\
\hline ES & Cax. & 14 & - & - & - & 14 & 1.900 & & 80 \\
\hline BS & S.M. & 21 & - & - & - & 21 & 2.230 & 500 & \\
\hline Tota & & 2870 & 159 & 1415 & 400 & 4844 & 602.248 & 25.755 & 1.975 \\
\hline
\end{tabular}

Fonte: ABEn. 
OGUISSO, T. - Relatório da tesouraria - período de 1978/1979. Rev. Bras. Enf; DF, 32 : 438-446, 1979.

\section{ANEXO IV}

QUADRO DEMONSTRATIVO DE BALANÇOS E BALA'NCETES MENSAIS RECEBIDOS (S) E NAO RECEBIDOS (N) DAS SECOES E DISTRITOS DE JULHO DE 1978 A JUNHO DE 1979

\begin{tabular}{|c|c|c|c|c|c|c|c|c|c|c|c|c|c|c|c|c|}
\hline \multirow{2}{*}{$\begin{array}{l}\text { Se } \\
\text { şäo }\end{array}$} & \multirow{2}{*}{$\begin{array}{l}\text { Dis } \\
\text { tri } \\
\text { to }\end{array}$} & \multirow{2}{*}{$\begin{array}{l}\text { Ano } \\
\text { Més }\end{array}$} & \multirow[b]{2}{*}{7} & \multirow[b]{2}{*}{8} & \multirow[b]{2}{*}{9} & \multirow[b]{2}{*}{10} & \multicolumn{2}{|c|}{1978} & \multirow[b]{2}{*}{$\boldsymbol{A}$} & \multirow[b]{2}{*}{ B } & \multirow[b]{2}{*}{1} & \multicolumn{2}{|c|}{1979} & \multirow[b]{2}{*}{4} & \multirow[b]{2}{*}{5} & \multirow[b]{2}{*}{6} \\
\hline & & & & & & & 11 & 12 & & & & 2 & 3 & & & \\
\hline$A M$ & & & $\mathbf{N}$ & $\mathbf{N}$ & $\mathbf{s}$ & $\mathbf{s}$ & $\mathbf{S}$ & $\mathbf{s}$ & $\mathbf{N}$ & $\mathbf{N}$ & $\mathbf{S}$ & $\mathbf{S}$ & $\mathbf{S}$ & $\mathbf{S}$ & $\mathbf{s}$ & $\mathbf{S}$ \\
\hline $\mathbf{P A}$ & & & $\mathbf{S}$ & $\mathbf{S}$ & $\mathbf{S}$ & $\mathbf{s}$ & $\mathbf{s}$ & $\mathbf{s}$ & $\mathbf{s}$ & $\mathbf{s}$ & $\mathbf{s}$ &.$s$ & $\mathbf{s}$ & $\mathbf{N}$ & $\mathbf{N}$ & $\mathbf{N}$ \\
\hline$C E$ & & & $\mathbf{S}$ & $\mathbf{s}$ & $\mathbf{s}$ & $\mathbf{s}$ & $\mathbf{s}$ & $\mathbf{s}$ & $\mathbf{s}$ & $\mathbf{S}$ & $\mathbf{S}$ & $\mathbf{s}$ & $\mathbf{S}$ & $\mathbf{s}$ & $\mathbf{S}$ & $\mathbf{S}$ \\
\hline HA & & & $\mathbf{s}$ & $\mathbf{s}$ & $\mathbf{S}$ & $\mathbf{s}$ & $\mathbf{S}$ & $\mathbf{S}$ & $\mathbf{s}$ & $\mathbf{s}$ & $\mathbf{S}$ & $\mathbf{s}$ & $\mathbf{S}$ & $\mathbf{s}$ & $\mathbf{S}$ & $\mathbf{s}$ \\
\hline$P I$ & & & $\mathbf{S}$ & $\mathbf{S}$ & $\mathbf{S}$ & $\mathbf{S}$ & $\mathbf{S}$ & $\mathbf{S}$ & $\mathbf{S}$ & $\mathbf{s}$ & $\mathbf{S}$ & $\mathbf{s}$ & $\mathbf{S}$ & $\mathbf{s}$ & $\mathbf{S}$ & $\mathbf{N}$ \\
\hline RN & & & $\mathbf{N}$ & $\mathbf{N}$ & $\mathbf{s}$ & $\mathbf{S}$ & $\mathbf{s}$ & $\mathbf{S}$ & $\mathbf{N}$ & $\mathbf{s}$ & $\mathbf{S}$ & $\mathbf{s}$ & $\mathbf{S}$ & $\mathbf{N}$ & $\mathbf{N}$ & $\mathbf{N}$ \\
\hline$P E$ & & & $\mathbf{N}$ & $\mathbf{N}$ & $\mathbf{N}$ & $\mathbf{N}$ & $\mathbf{N}$ & $\mathbf{N}$ & $\mathbf{N}$ & $\mathbf{N}$ & $\mathbf{N}$ & $\mathbf{N}$ & $\mathbf{N}$ & $\mathbf{N}$ & $\mathbf{N}$ & $\mathbf{N}$ \\
\hline P B & & & $\mathbf{S}$ & $\mathbf{S}$ & $\mathbf{s}$ & $\mathbf{s}$ & $\mathbf{S}$ & $\mathbf{S}$ & $\mathbf{N}$ & $\mathbf{N}$ & $\mathbf{S}$ & $\mathbf{s}$ & $\mathbf{S}$ & $\mathbf{s}$ & $\mathbf{S}$ & $\mathbf{S}$ \\
\hline A L & & & $\mathbf{S}$ & $\mathbf{S}$ & $\mathbf{s}$ & $\mathbf{N}$ & $\mathbf{N}$ & $\mathbf{N}$ & $\mathbf{N}$ & $\mathbf{N}$ & $\mathbf{N}$ & $\mathbf{N}$ & $\mathbf{N}$ & $\mathbf{N}$ & $\mathbf{N}$ & $\mathbf{N}$ \\
\hline$S E$ & & & $\mathbf{S}$ & $\mathbf{S}$ & $\mathbf{S}$ & $\mathbf{s}$ & $\mathbf{s}$ & $\mathbf{s}$ & $\mathbf{s}$ & $\mathbf{N}$ & $\mathbf{s}$ & $\mathbf{s}$ & $\mathbf{S}$ & $\mathbf{s}$ & $\mathbf{s}$ & $\mathbf{S}$ \\
\hline $\mathbf{B A}$ & & & $\mathbf{S}$ & $\mathbf{s}$ & $\mathbf{s}$ & $\mathbf{s}$ & $\mathbf{S}$ & $\mathbf{s}$ & $\mathbf{s}$ & $\mathbf{S}$ & $\mathbf{S}$ & $\mathbf{s}$ & $\mathbf{s}$ & $\mathbf{s}$ & $\mathbf{S}$ & $\mathbf{S}$ \\
\hline MT & & & $\mathbf{S}$ & $\mathbf{S}$ & $\mathbf{S}$ & $\mathbf{S}$ & $\mathbf{S}$ & $\mathbf{S}$ & $\mathbf{S}$ & $\mathbf{S}$ & $\mathbf{S}$ & $\mathbf{S}$ & $\mathbf{S}$ & $\mathbf{s}$ & $\mathbf{S}$ & $\mathbf{S}$ \\
\hline MG & В.н. & & $\mathbf{S}$ & s & s & $\mathbf{S}$ & $\mathbf{S}$ & $\mathbf{s}$ & $\mathbf{s}$ & $\mathbf{N}$ & $\mathbf{S}$ & s & $\mathbf{s}$ & $\mathbf{s}$ & $\mathbf{S}$ & $\mathbf{S}$ \\
\hline MG & $\mathbf{J} \cdot \mathbf{F}$ & & $\mathbf{N}$ & $\mathbf{N}$ & $\mathbf{N}$ & $\mathbf{N}$ & $\mathbf{N}$ & $\mathbf{N}$ & $\mathbf{s}$ & $\mathbf{N}$ & $\mathbf{N}$ & $\mathbf{N}$ & $\mathbf{N}$ & $\mathbf{N}$ & $\mathrm{N}$ & $\mathbf{N}$ \\
\hline GO & Goi. & & $\mathbf{S}$ & $\mathbf{S}$ & $\mathbf{S}$ & $\mathbf{S}$ & $\mathbf{S}$ & $\mathbf{S}$ & $\mathbf{s}$ & $\mathbf{s}$ & $\mathbf{N}$ & $\mathbf{N}$ & $\mathbf{N}$ & $\mathbf{N}$ & $\mathbf{N}$ & $\mathbf{N}$ \\
\hline GO & R.V. & & $\mathbf{N}$ & $\mathbf{N}$ & $\mathbf{N}$ & $\mathbf{N}$ & $\mathbf{N}$ & $\mathbf{N}$ & $\mathbf{N}$ & $\mathbf{N}$ & $\mathbf{N}$ & $\mathbf{N}$ & $\mathbf{N}$ & $\mathbf{N}$ & $\mathbf{N}$ & $\mathbf{N}$ \\
\hline$D F$ & & & $\mathbf{S}$ & $\mathbf{s}$ & $\mathbf{s}$ & $\mathbf{s}$ & $\mathbf{s}$ & $\mathbf{s}$ & $\mathbf{S}$ & $\mathbf{S}$ & $\mathbf{s}$ & $\mathbf{S}$ & $\mathbf{S}$ & $\mathbf{S}$ & $\mathbf{N}$ & $\mathbf{N}$ \\
\hline ES & & & $\mathbf{N}$ & $\mathbf{N}$ & $\mathbf{N}$ & $\mathbf{N}$ & $\mathbf{N}$ & $\mathbf{N}$ & $\mathbf{N}$ & $\mathbf{N}$ & $\mathbf{N}$ & $\mathbf{N}$ & $\mathbf{N}$ & $\mathbf{N}$ & $\mathbf{N}$ & $\mathbf{N}$ \\
\hline$\dot{R} \mathrm{~J}$ & RJ. & & $\mathbf{s}$ & $\mathbf{N}$ & $\mathbf{s}$ & $\mathbf{s}$ & $\mathbf{S}$ & $\mathbf{s}$ & $\mathbf{s}$ & $\mathbf{s}$ & $\mathbf{s}$ & $\mathbf{s}$ & $\mathbf{s}$ & $\mathbf{s}$ & $\mathbf{s}$ & $\mathbf{s}$ \\
\hline$R J$ & Nit. & & $\mathbf{S}$ & $\mathbf{s}$ & $\mathbf{s}$ & $\mathbf{N}$ & $\mathbf{N}$ & $\mathbf{N}$ & $\mathbf{s}$ & $\mathbf{N}$ & $\mathbf{S}$ & $\mathbf{s}$ & $\mathbf{S}$ & $\mathbf{N}$ & $\mathbf{N}$ & $\mathbf{N}$ \\
\hline $\mathrm{RJ}$ & V.R. & & $\mathbf{N}$ & $\mathbf{N}$ & $\mathbf{N}$ & $\mathbf{N}$ & $\mathbf{N}$ & $\mathbf{N}$ & $\mathbf{N}$ & $\mathbf{N}$ & $\mathbf{N}$ & $\mathbf{N}$ & $N$ & $\mathbf{N}$ & $\mathbf{N}$ & $\mathbf{N}$ \\
\hline SP & SP. & & $\mathbf{S}$ & $\mathbf{S}$ & S. & $\mathbf{S}$ & $\mathbf{S}$ & $\mathbf{S}$ & $\mathbf{S}$ & $\mathbf{S}$ & $\mathbf{S}$ & $\mathbf{S}$ & S " & $\mathbf{S}$ & $\mathbf{S}$ & $\mathbf{N}$ \\
\hline$S P$ & San. & & $\mathbf{N}$ & $\mathbf{N}$ & $\mathbf{N}$ & $\mathbf{N}$ & $\mathbf{N}$ & $\mathbf{N}$ & $\mathbf{N}$ & $\mathbf{N}$ & $\mathbf{N}$ & $\mathbf{N}$ & $\mathbf{N}$ & $\mathbf{N}$ & $\mathrm{N}$ & $\mathbf{N}$ \\
\hline SP & R.P. & & $\mathbf{N}$ & $\mathbf{N}$ & $\mathbf{N}$ & $\mathbf{N}$ & $\mathbf{N}$ & $\mathbf{N}$ & $\mathbf{S}$ & $\mathbf{N}$ & $\mathbf{N}$ & $\mathbf{N}$ & $\mathbf{N}$ & $\mathbf{N}$ & N & $\mathbf{N}$ \\
\hline SP & $C a m p$ & & $\mathbf{N}$ & $\mathbf{N}$ & $\mathbf{N}$ & $\mathbf{N}$ & $\mathbf{N}$ & $\mathbf{N}$ & $\mathbf{s}$ & $\mathbf{s}$ & $\mathbf{N}$ & $\mathbf{N}$ & $\mathbf{N}$ & $\mathbf{N}$ & $\mathbf{N}$ & $\mathbf{N}$ \\
\hline SP & Arar & & $\mathbf{N}$ & $\mathbf{N}$ & $\mathbf{N}$ & $N$ & $\mathbf{N}$ & $\mathbf{N}$ & $\mathbf{S}$ & $\mathbf{N}$ & $\mathbf{N}$ & $\mathbf{N}$ & $\mathrm{N}$ & $\mathbf{N}$ & $\mathbf{N}$ & $\mathbf{N}$ \\
\hline$P R$ & Cur. & & $\mathbf{N}$ & $N$ & $\mathbf{N}$ & $\mathbf{N}$ & $\mathbf{N}$ & $\mathbf{S}$ & $\mathbf{S}$ & $\mathbf{N}$ & $\mathbf{S}$ & $\mathbf{s}$ & $\mathbf{S}$ & $\mathbf{S}$ & $\mathbf{S}$ & $\mathbf{S}$ \\
\hline$P \mathbf{R}$ & Lon & & $\mathbf{N}$ & $\mathbf{N}$ & $\mathbf{N}$ & $\mathbf{N}$ & $\mathbf{N}$ & $\mathbf{N}$ & $\mathbf{N}$ & $\mathbf{N}$ & $\mathbf{N}$ & $\mathbf{N}$ & $\mathbf{N}$ & $\mathbf{N}$ & $\mathbf{N}$ & $\mathbf{N}$ \\
\hline SC & & & $\mathbf{N}$ & $\mathbf{N}$ & $\mathbf{N}$ & $\mathbf{N}$ & $\mathbf{N}$ & $\mathbf{N}$ & $\mathbf{N}$ & $\mathbf{N}$ & $\mathbf{N}$ & $\mathbf{N}$ & $\mathbf{N}$ & $\mathbf{N}$ & $\mathbf{N}$ & $\mathbf{N}$ \\
\hline R S & P.A. & & $\mathbf{S}$ & $\mathbf{N}$ & $\mathbf{s}$ & $\mathbf{N}$ & $\mathbf{N}$ & $\mathbf{N}$ & $\mathbf{S}$ & $\mathbf{N}$ & $\mathbf{s}$ & $\mathbf{s}$ & $\mathbf{S}$ & $\mathbf{S}$ & $\mathbf{S}$ & $\mathbf{S}$ \\
\hline R.S & $\operatorname{Cax}$ & & $\mathbf{N}$ & $\mathbf{N}$ & $\mathbf{N}$ & $\mathbf{N}$ & $\mathbf{N}$ & $\mathbf{N}$ & $\mathbf{N}$ & $\mathbf{N}$ & $\mathbf{N}$ & $\mathbf{N}$ & $\mathbf{N}$ & $\mathbf{N}$ & $\mathbf{N}$ & $\mathbf{N}$ \\
\hline RS & $\mathrm{S} . \mathrm{Ma}$ & & $\mathbf{S}$ & $\mathbf{s}$ & $\mathbf{s}$ & $\mathbf{N}$ & $\mathbf{N}$ & $\mathbf{N}$ & $\mathbf{S}$ & $\mathbf{N}$ & $\mathbf{N}$ & $\mathbf{N}$ & $\mathbf{S}$ & $\mathbf{s}$ & $S$ & $\mathbf{S}$ \\
\hline
\end{tabular}

A - Demonstrativo da Receita e Despesas de janeiro a dezembro de 1978.

B - Balanço Patrimonial de janeiro a dezembro de 1978. 